convulsion length was 7.4 seconds $(95 \% \mathrm{CI}, 3.7$ to 11.1 seconds) or $34 \%(95 \% \mathrm{CI}, 17$ to $51 \%)$.

The present findings support the hypothesis and hence the supposition that the anticonvulsant as well as anaesthetic effects of methohexitone decline rapidly after bolus injection. The implications for the practice and theory of ECT require further investigation, but the findings ought to reemphasise how important anaesthetic technique is in the practice of ECT. An increased delay between induction and electrical stimulation may be of value in some patients as a technique for seizure augmentation, that is, when a patient fails to have an adequate convulsion after stimulation with the maximum output of an ECT machine.

AdDerSLEY, D. J. \& Hammton, M. (1953) Use of succinylcholine in ECT. British Medical Journal, 1, 195-197.

AyD, F. J. (1961) Methohexital (Brevital): A new anaesthetic for electroconvulsant therapy. Diseases of the Nervous System, 22. 388-390.

Corssen, G., Raves, J. G. \& Stanley, T. H. (1988) Intravenou Anaesthesia and Analgesia. Philadelphia: Lea \& Febiger.

I. P. Collins

I. F. SCOTT

Royal Edinburgh Hospital

Edinburgh EHIO $5 \mathrm{HF}$

\section{Counselling and community psychiatric nurses}

SIR: There is an urgent need for studies of the efficacy of counselling in primary medical care, and a need to establish the most effective deployment of community psychiatric nurses (CPNs). While Gournay \& Brooking's study (BJP, February 1994, 164, 231-238) was welcome, few conclusions can be drawn from the results due to a design failure.

The data for the two samples of patients who were randomised differently were merged at the six month follow-up point. This is inappropriate as the patients who were kept on a waiting list before entering counselling had received less therapy at follow-up. Controlled trials in general practice are difficult to do (Tognoni et al, 1991) and careful piloting is required before embarking on a large investigation (King et al, 1994).

The figures given in the paper are confusing. According to the flow diagram 106 patients were randomised to CPN care, whereas in the text this number becomes 92 . Whether drop out occurred from CPN care, from the research assessments or both is not clear. It is stated that $50 \%$ of patients randomised to CPN care dropped out. We realise it is difficult to measure GP care, however, only $55 \%$ of patients randomised to the GP completed the third research assessment. No intention to treat analysis appears to have been carried out, even when the data for the practice consultation rates for all patients entering the trial must have been available. No attempt was made to determine the type of intervention carried out by the CPNs.

Despite these limitations, Gournay \& Brooking (1994) conclude that CPNs are ineffective as counsellors in general practice and would be more appropriately deployed in the care of the severely mentally ill. We sympathise with the difficulties of conducting good controlled research of brief psychotherapies in general practice. Unfortunately these data simply do not bear out the authors' sweeping conclusions.

King, M., Broster, G., Lloyd, M., et al (1994) Controlled trials in the evaluation of counselling in general practice. British Journal of General Practice, 44, 229-232.

Tognoni, G., Alli, C., Avanzini, F., et al (1991) Randomised clinical trials in general practice: lessons from a failure. British Medical Journal, 303, 969-971.

K. FRIEDLI
M. KING
London Free Hospital School of Medicine

AuthoR's RePLy: We are grateful to Friedli \& King for drawing attention to the apparent discrepancies in the text. The flow diagram shows 106 randomised to CPN care and the figure in the text of 92 refers to the number of patients who actually took up the offer of this intervention. We can confirm that $50 \%$ of patients who commenced CPN intervention dropped out.

With regard to our comparison of patients $r$ : ceiving CPN interventions and patients attending their GP, we only used patients who had been assigned to immediate CPN intervention in this analysis - we omitted the group of eight patients who were still in CPN treatment after a period on the waiting list. Thus, our comparison involved patients allocated to exactly the same conditions of treatment. Friedl $\&$ King are incorrect in their assumptions, (a) that there was no intention to treat analysis carried out; or (b) that we made no attempt to determine the type of interventions carried out by CPNs. We videotaped a selection of assessment interviews and collected a considerable amount of other data including CPNs' intention for treatment. The results of some of these data are reported elsewhere (Gournay et al, 1993), and further analyses of the process data are being carried out. It must be 